\title{
REMARKS ON CAUCHY'S INTEGRAL FORMULA IN MATRIC SPACES
}

\author{
JOSEPHINE MITCHELL
}

1. Introduction. Recently several proofs of Cauchy's integral formula have been given for matric spaces $[2 ; 4 ; 5 ; 7]$. However a short direct proof is available by using the argument that Morita gives to prove the Poisson formula (see \$2). In $\$ 3$ the formula is also proved by means of a minimal problem, similar to those introduced by Bergman [1]. Since the present paper is closely related to Morita's [7], we use his notation wherever possible.

The matric spaces under consideration are the four main types of irreducible bounded symmetric domains given by E. Cartan [3]. Let $z$ be a matrix of complex numbers, $z^{\prime}$ its transpose, $z^{*}$ its conjugate transpose and $I^{(r)}$ the identity matrix of order $r$. Then the first three types are defined by

$$
D=E\left[z \mid I^{(n)}-z^{*} z>0\right],
$$

where

I. $\mathfrak{A}_{m n}: z$ is a matrix of type $(m, n)(m \geqq n)$.

II. $\mathfrak{S}_{n}: z$ is a symmetric matrix of order $n$.

III. $R_{n}: z$ is a skew symmetric matrix of order $n$.

The fourth type is

IV. $\mathfrak{M}_{n}$ : the set of all matrices $z$ of type $(n, 1)$ (that is, $n$-dimensional vectors) such that

$$
\left|z^{\prime} z\right|<1, \quad 1-2 z^{*} z+\left|z^{\prime} z\right|^{2}>0 .
$$

It is known that each of the domains possesses a distinguished boundary $B[1]$, which is defined by

$$
z^{*} z=I^{(n)}
$$

for $\mathfrak{A}_{m n}, \mathfrak{S}_{n}$ and for $\mathfrak{R}_{n}$ if $n$ is even, or the eigenvalues of $z^{*} z$ are all 1 except one which is zero if $n$ is odd. For $\mathfrak{M}_{n}, B$ is given by

$$
z^{*} z=1, \quad\left|z^{\prime} z\right|=1 .
$$

2. Cauchy's integral formula. We define a kernel function (the Cauchy kernel) by

$$
K(z, \zeta)=V^{-1} \operatorname{det}^{-p}(z-\zeta),
$$

Received by the editors March 4, 1959 and, in revised form, May 11, 1959. 
where $p=n$ for domains $\mathfrak{A}_{n n},(n+1) / 2$ for $\mathfrak{S}_{n},(n-1) / 2$ for $\mathfrak{R}_{n}$ if $n$ is even, and $V$ is the Euclidean volume of the domain $B$. For domains $\mathfrak{M}_{n}$

$$
K(z, \zeta)=V^{-1}\left[(z-\zeta)^{\prime}(z-\zeta)\right]^{-n / 2}
$$

Then

TheOREM 1. Let $f(z)$ be regular in $D$ and continuous on $\bar{D}$ (the closure of $D$ ), where $D$ is one of the domains $\mathfrak{A}_{n n}, \mathfrak{S}_{n}, \mathfrak{R}_{2 n}$ or $\mathfrak{M}_{n}$. Then

$$
f(\zeta)=\int_{B} K(\zeta, z) f(z) \dot{z}, \quad \zeta \in D,
$$

where

$$
\begin{array}{rlr}
\dot{z} & =c_{n 1} \prod_{j, k=1}^{n} d z_{j k} & \text { for } \mathfrak{A}_{n n} \\
& =c_{n 2} \prod_{j=1 ; k \geq j}^{n} d z_{j k} & \text { for } \mathfrak{S}_{n} \\
& =c_{n 3} \prod_{j=1 ; k>j}^{n-1} d z_{j k} & \text { for } \mathfrak{\&}_{n}(n \text { even }) \\
& =c_{n 4} \prod_{j=1}^{n} d z_{j} & \text { for } \mathfrak{M}_{n},
\end{array}
$$

and the constants $c_{n j}$ are such that $V^{-1} \int_{B} K^{-1}(z, 0) \dot{z}=1$ in each case. (We note that $K^{-1}(z, 0) \dot{z}$ is the Euclidean volume element for the set $B$ [7].)

Proof. We shall restrict ourselves to the case $D=\mathfrak{A}_{n n}$ but the other cases may be treated similarly. See $[7, \S 16]$ for the details in the case $\mathfrak{M}_{n}$.

It is known that the set of analytic mappings taking $D$ onto itself and $\zeta$ into 0 and the inverse transformations are given by [8]

$$
\begin{aligned}
& w=a(z-\zeta)\left(d-d \zeta^{*} z\right)^{-1} \\
& \begin{aligned}
z=\sigma(w) & =\left(a+w d \zeta^{*}\right)^{-1}(w d+a \zeta) \\
& =\left(a^{*} w+\zeta d^{*}\right)\left(d^{*}+\zeta^{*} a^{*} w\right)^{-1}
\end{aligned}
\end{aligned}
$$

subject to the conditions

$$
\begin{aligned}
& a\left(I-\zeta \zeta^{*}\right) a^{*}=I, \quad d\left(I-\zeta^{*} \zeta\right) d^{*}=I, \quad \zeta^{*} a^{*} a=d^{*} d \zeta^{*}, \\
& a^{*} a-\zeta d^{*} d \zeta^{*}=I, \quad d^{*} d-\zeta^{*} a^{*} a \zeta=I,
\end{aligned}
$$

$\left(I=I^{(n)}\right)$. Also these transformations leave the set $B$ invariant. 
Suppose first that $f(z)$ is analytic on $\bar{D}$ and consider the expression

$$
F\left(\zeta, z, z^{*}, \dot{z}\right)=f(z) \operatorname{det}^{-n}\left(I-z^{*} \zeta\right) d v_{z},
$$

where $d v_{z}$ is the Euclidean volume element of the set $B$ :

$$
d v_{z}=\operatorname{det}^{-n} z \dot{z} .
$$

Under the transformation (9)

but [6]

$$
\dot{z}=\frac{\partial(z)}{\partial(w)} \dot{w}
$$

$$
\frac{\partial(z)}{\partial(w)}=\operatorname{det}^{-n}\left(a+w d \zeta^{*}\right) \operatorname{det}^{-n}\left(d^{*}+\zeta^{*} a^{*} w\right)
$$

Also since $w^{*} w=I$,

$$
d v_{z}=\operatorname{det}^{-n}\left(d+w^{*} a \zeta\right) \operatorname{det}^{-n}\left(d^{*}+\zeta^{*} a^{*} w\right) d v_{w},
$$

and

$$
I-z^{*} \zeta=\left(d+w^{*} a \zeta\right)^{-1} d\left(I-\zeta^{*} \zeta\right)
$$

Thus

$$
\int_{B} F\left(\zeta, z, z^{*}, \dot{z}\right)=\operatorname{det}^{-n} d \operatorname{det}^{-n}\left(I-\zeta^{*} \zeta\right) \int_{B} f_{0}(w) d v_{w} .
$$

where

$$
f_{0}(w)=\operatorname{det}^{-n}\left(d^{*}+\zeta^{*} a^{*} w\right) f(\sigma(w))
$$

is regular on $\bar{D}$.

By a theorem due to H. Cartan a regular function on $\bar{D}$ can be expanded on $\bar{D}$ into a uniformly convergent series of homogeneous polynomials, $\sum_{n=0}^{\infty} a_{n} P_{n}(w)$, where $P_{0}(w)$ is a constant so that $a_{0} P_{0}=f_{0}(0)$ and $P_{n}$ is of degree $>0$ for $n>0$. Also since $B$ is circular [5]

$$
\int_{B} P_{n}(w) d v_{w}=0 \quad \text { for } n>0
$$

Thus

$$
\int_{B} f_{0}(w) d v_{w}=V f_{0}(0)
$$

and by (9a) and (10) 


$$
(1 / V) \int_{B} F\left(\zeta, z, z^{*}, \dot{z}\right)=f(\zeta)
$$

Since on $z^{*} z=I$,

$$
\operatorname{det}^{-n}\left(I-z^{*} \zeta\right) d v_{z}=\operatorname{det}^{-n}(z-\zeta) \dot{z},
$$

(7) follows for functions regular on $\bar{D}$.

In case $f(z)$ is regular on $D$ and continuous on $\bar{D}$, following Morita, we have

$$
f(t \zeta)=\int_{B} K(\zeta, z) f(t z) d v_{z}
$$

for any real number $t$ such that $0 \leqq t<1$. Letting $t \rightarrow 1^{-}$we see that (7) holds for such a function $f(z)$. Thus Theorem 1 is proved.

3. Minimal problem. Let $D$ be an arbitrary bounded domain in the space of $n$ complex variables with a distinguished boundary $B$. Let $\zeta$ be an arbitrary fixed point of $D$ and consider the subclass $S$ of regular functions $f$ on $\bar{D}$ such that $f(\zeta)=1$. Suppose there exists a function $M(\zeta, z)$ of $S$ which minimizes the integral

$$
\int_{B}|f(z)|^{2} d v_{z}, \quad \in S \text {. }
$$

Then defining

$$
K_{0}^{*}(\zeta, z)=M(\zeta, z) / \int_{B} M(\zeta, w) d v_{w}
$$

we have

$$
\frac{K_{0}^{*}(\zeta, z)}{K_{0}^{*}(\zeta, \zeta)}=\frac{M(\zeta, z)}{\int_{B} M(\zeta, w) d v_{w}} \cdot \int_{B} \frac{M(\zeta, w) d v_{w}}{M(\zeta, \zeta)}=M(\zeta, z)
$$

In analogy to the case of one complex variable we call the function $K_{0}(\zeta, z)$ the Szegö kernel of the domain $D$.

THEOREM 2. Let $M(\zeta, z)$ be a solution of the above minimal problem and $K_{0}(\zeta, z)$ the kernel defined by (11). Then for any $f$ regular on $\bar{D}$ the (reproducing) formula

$$
f(\zeta)=\int_{B} K_{0}(\zeta, z) f(z) d v_{z}, \quad \zeta \in D .
$$

holds. Also the minimum value of the integral is $\left[1 / K_{0}(\zeta, \zeta)\right]$. 
Proof. From the minimal property for any arbitrary complex $\epsilon$ and regular $f$

$$
\int_{B}|M|^{2} d v \leqq \int_{B}|M+\epsilon[f(z)-f(\zeta)]|^{2} d v .
$$

Thus

$$
2 \operatorname{Re}\left[\epsilon \int_{B} M^{*}[f(z)-f(\zeta)] d v\right]+|\epsilon|^{2} \int_{B}|f(z)-f(\zeta)|^{2} d v \geqq 0 .
$$

Since $|\epsilon|$ and arg $\epsilon$ are both arbitrary, it follows that

$$
\int_{B} M^{*}(\zeta, z)[f(z)-f(\zeta)] d v=0
$$

from which (12) results. Also from (12)

$$
\begin{aligned}
\int_{B}|M(\zeta, z)|^{2} d v & =\left|K_{0}^{-2}(\zeta, \zeta)\right| \int_{B} K_{0}(\zeta, z) K_{0}^{*}(\zeta, z) d v \\
& =K_{0}^{-1}(\zeta, \zeta) .
\end{aligned}
$$

For the matric spaces as we have seen in $\$ 2$ this formula is valid for any $f$ regular on $D$ and continuous on $\bar{D}$.

For the domains $\mathfrak{A}_{n n}, \mathfrak{S}_{n}$ and $\mathfrak{R}_{2 m}$ the kernel $K_{0}(\zeta, z)$ is equal to

$$
K_{0}(\zeta, z)=V^{-1} \operatorname{det}^{-p}\left(I-z^{*} \zeta\right),
$$

which equals $\operatorname{det}^{p} z K(\zeta, z)$ if $z \in B$, where $p$ has the same values as for the kernel $K(\zeta, z)$; for $\mathfrak{M}_{n}$

$$
K_{0}(\xi, z)=V^{-1}\left[1-2 z^{*} \zeta+\left(\zeta^{\prime} \zeta\right)\left(z^{\prime} z\right)^{*}\right]^{-n / 2} .
$$

The proof that the minimal problem has a solution for the matric spaces and that (13) satisfies (11) is similar to that in [6] for the Bergman kernel function and will be omitted here.

\section{REFERENCES}

1. S. Bergman, The kernel function and conformal mapping, Mathematical Surveys, no. 5, 1950.

2. S. Bochner, Group invariance of Cauchy's formula in several variables, Ann. of Math. vol. 45 (1944) pp. 686-707.

3. E. Cartan, Sur les domains bornés homogènes de l'espace de $n$ variables complexes, Abh. Math. Sem. Univ. Hamburg vol. 11 (1936) pp. 116-162.

4. L. K. Hua, On the theory of functions of several complex variables, I-III (in Chinese), Acta Math. Sinica vol. 2 (1953) pp. 288-323; vol. 5 (1955) pp. 1-25 and pp. 205-242. 
5. - Harmonic analysis of the classical domains in the study of analytic functions of several complex variables, mimeographed notes, about 1956.

6. J. Mitchell, The kernel function in the geometry of matrices, Duke Math. J. vol. 19 (1952) pp. 575-584.

7. K. Morita, On the kernel functions of symmetric domains, Science Reports of the Tokyo Kyoiku Daigaku, Section A vol. 5 (1956) pp. 190-212.

8. C. L. Siegel, Analytic functions of several complex variables, Notes by P. T. Bateman, Institute for Advanced Study, Princeton, 1948-1949.

The Pennsylvania State University

\section{A COUNTABLE INTERPOLATION PROBLEM}

\section{Z. A. MELZAK}

1. Let $\mathfrak{H C}$ be the set of all order-preserving homeomorphisms of $I=[0,1]$ onto itself. $\mathcal{H}$ is a metric space in the uniform metric $\rho$ :

$$
\rho\left(f_{1}, f_{2}\right)=\max _{I}\left|f_{1}(x)-f_{2}(x)\right|, \quad f_{1}, f_{2} \in \mathcal{F C} .
$$

Franklin [1] has proved the following theorem: (A) Let $A$ and $B$ be two countable sets, each dense on $I$. Then the set of analytic $f \in \mathcal{H}$, such that $f(A)=B$, is dense in $\mathcal{H}$.

It follows from (A) and from its extension in [2] that there exist nontrivial analytic functions $f \in \mathcal{H}$, such that $f(x)$ is transcendental for each transcendental $x \in I$, and for each algebraic $x \in I, x$ and $f(x)$ are algebraic and of the same degree.

In this note, without using either of these results, we prove a similar but complementary statement by means of Baire's Category Theorem.

THEOREM 1. Let $\mathfrak{K}_{\alpha}, \alpha>2$, be the subset of $\mathfrak{F}$ consisting of all functions $f \in \mathcal{H}$, whose values are either rational or transcendental and approximable to degree $>\alpha$, for each algebraic $x \in I$. Then $\mathcal{K}_{\alpha}$ is a dense $G_{\delta}$-set of second category in $\mathcal{H}$.

2. Since $\mathcal{F}$ is not complete in $\rho$, we first remetrize it. Let

$$
\sigma\left(f_{1}, f_{2}\right)=\rho\left(f_{1}, f_{2}\right)+\rho\left(f_{1}^{-1}, f_{2}^{-1}\right), \quad f_{1}, f_{2} \in \mathcal{H} .
$$

Lemma 1. $\mathfrak{H}$ is complete in the $\sigma$-metric.

Let $\mathcal{F}=I^{I}$ be the set of all continuous maps from $I$ into $I$, then $\mathcal{F}$ is complete in $\rho$. Let $\left\{f_{n}\right\}, n=1,2, \cdots$, be a $\sigma$-Cauchy sequence in FC. Then $\left\{f_{n}\right\}$ is also a $\rho$-Cauchy sequence in $\mathcal{F}$, therefore $f_{n} \rightarrow f$,

Received by the editors May 8, 1959 and, in revised form, May 25, 1959. 